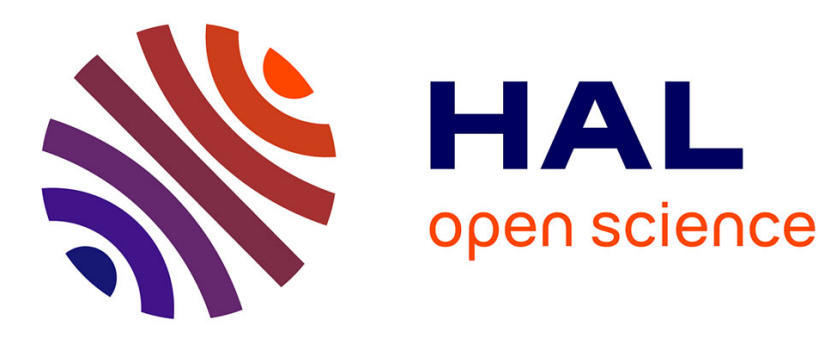

\title{
A new real space renormalisation method and its Julia set
}

\author{
Bernard Derrida, H Flyvbjerg
}

\section{To cite this version:}

Bernard Derrida, H Flyvbjerg. A new real space renormalisation method and its Julia set. Journal of Physics A : Mathematical and General, 1985, 18 (6), pp.L313-L318. 10.1088/0305-4470/18/6/010 . hal-03285943

\section{HAL Id: hal-03285943 \\ https://hal.science/hal-03285943}

Submitted on 19 Jul 2021

HAL is a multi-disciplinary open access archive for the deposit and dissemination of scientific research documents, whether they are published or not. The documents may come from teaching and research institutions in France or abroad, or from public or private research centers.
L'archive ouverte pluridisciplinaire HAL, est destinée au dépôt et à la diffusion de documents scientifiques de niveau recherche, publiés ou non, émanant des établissements d'enseignement et de recherche français ou étrangers, des laboratoires publics ou privés. 


\title{
LETTER TO THE EDITOR
}

\section{A new real space renormalisation method and its Julia set}

\author{
B Derrida and H Flyvbjerg \\ Service de Physique Théorique, CEN Saclay 91191 Gif-sur-Yvette Cedex, France
}

Received 14 January 1985

\begin{abstract}
We introduce a new real space renormalisation method which is valid not only on the real axis but also everywhere in the complex plane of temperatures. The method requires the knowledge of the partition function on small lattices and can in principle be improved systematically. We test this method on the two-dimensional Ising model. We find that it gives a satisfactory picture for the set of zeros of the partition function. When restricted to the real axis, the estimates for the renormalisation transformation, the critical point and the thermal exponent are also very good.
\end{abstract}

Real space renormalisation methods (Burkhardt and van Leeuwen 1982 and references therein) have become a very useful way of understanding the phase diagrams and the critical properties of models of statistical mechanics. Some of them are very simple but not very accurate (like the Migdal-Kadanoff method). Others (like the Kadanoff variational method) are more sophisticated and give in a lot of cases good estimates of the critical exponents. Yet others (like the phenomenological renormalisation or the Monte Carlo renormalisation based on block spin calculations) can in principle be improved in a systematic way. Thus there exist at present several satisfactory methods to calculate the critical properties of sufficiently simple models like the Ising model. However, with these methods it is difficult to study the whole set of singularities of the free energy in the complex plane of temperatures. In order to justify this assertion we discuss these methods one at a time: the Monte Carlo method is very well suited for complex probabilities. The phenomenological renormalisation (Nightingale 1982) which requires the calculation of the two largest eigenvalues of the transfer matrix suffers from the difficulty that when the temperature moves to the complex plane, the eigenvalues may cross and therefore the renormalisation transformation or its derivative is not defined along the lines where the eigenvalues cross. Block spins methods are also well adapted to describe the ferromagnetic transition but need modification to describe antiferromagnetic order and therefore has little chance to work everywhere in the complex plane.

Recently it has been shown that on hierarchical lattices, for which one can write an exact real space renormalisation transformation, the knowledge of the real space renormalisation was sufficient to produce the whole set of zeros of the partition function: the result was that the Julia set of the renormalisation transformation is the accumulation set of the zeros of the partition function (Derrida et al 1983, Itzykson and Luck 1984, Peitgen et al 1984). One may then ask whether for usual Bravais lattices one can construct approximate real space renormalisations, the Julia sets of which approximate the entire set of zeros of the partition function in the complex plane. 
The purpose of the present letter is to give a positive answer to that question. Let us consider for example an Ising model on a $d$-dimensional lattice. The Hamiltonian of this Ising model is

$$
\mathscr{H}=-J \sum_{\langle i j\rangle} S_{i} S_{j}
$$

where there is a spin $S_{i}= \pm 1$ on each lattice site and the sum runs over the pairs of nearest neighbours on the lattice. Let us define $t$ as

$$
t=\tanh \beta J \text {. }
$$

The real space renormalisation method that we propose in order to produce the whole set of zeros as the Julia set of the renormalisation transformation is the following

$$
\frac{Z_{N}(t)}{\left[Z_{N / b^{d}}(t)\right]^{b^{d}}}=\frac{Z_{N / b^{d}}\left(t^{\prime}\right)}{\left[Z_{N / b^{2 d}}\left(t^{\prime}\right)\right]^{b^{d}}}
$$

where $Z_{N}$ is the partition function of a finite lattice of $N$ sites with periodic boundary conditions ( $N=l^{d}$ and $l$ is the linear dimension of the lattice). Equation (3) defines a transformation $t \rightarrow t^{\prime}$ associated with a change of scale $b$.

In this letter we shall first try to explain why (3) is a very plausible real space renormalisation. Then, we shall test the method on the two-dimensional Ising model on a square lattice. We shall first look at its predictions for the critical point and the critical exponent on the real axis. Then we shall look at the shape of the renormalisation transformation. Finally we shall give pictures of the Julia set associated with the renormalisation transformation (3) and compare it with the known exact results (Fisher 1964). The denomination 'Julia set' is slightly abusive here, since $t$ ' is a multi-valued function of $t$.

Let us first start by seeing how (3) can be derived. If one defines $f(t)$ as the free energy per site at temperature $t$, the result of any real space renormalisation $t \rightarrow t^{\prime}$ associated with a change of scale of ratio $b$ is to relate $f(t)$ to $f\left(t^{\prime}\right)$ by a formula of the following form

$$
f(t)=\left(1 / b^{d}\right) f\left(t^{\prime}\right)+g(t)
$$

where $g(t)$ can usually be calculated by the renormalisation procedure. If we multiply equation (4) by $N$ (the number of sites in the lattice) and if one takes the exponential, one ends up with the following formula

$$
Z_{N}(t)=Z_{N / b^{d}}\left(t^{\prime}\right) \exp (N g(t)) .
$$

What we want to do is to build a relationship between $t$ and $t^{\prime}$ from the knowledge of the partition functions $Z_{N}(t)$. Obviously this is not possible since we do not know a priori what function $g(t)$ to choose. We can avoid that difficulty by writing equation (3) twice and by eliminating the function $g(t)$. If one rewrites (5) as

$$
Z_{N / b^{d}}(t)=Z_{N / b^{2 d}}\left(t^{\prime}\right) \exp \left(N g(t) / b^{d}\right)
$$

one sees easily that by taking the ratio of (5) and (6) taken to the power $b^{d}$, one can eliminate the function $g(t)$ and one ends up with the renormalisation formula (3).

At this stage it is useful to make an important comment: formula (3) works only because of finite size effects. In some sense, it is a finite size scaling formula. Let us restrict ourselves for the moment to the case of real temperatures. We write (3) in the 
following way

$$
f_{N}(t)-f_{N / b^{d}}(t)=\left(1 / b^{d}\right)\left[f_{N / b^{d}}\left(t^{\prime}\right)-f_{N / b^{2 d}}\left(t^{\prime}\right)\right]
$$

where $f_{N}(t)$ is the free energy per site of a finite lattice of $N=l^{d}$ sites. Finite size scaling (Barber 1983) tells us that the free energy $f_{N}$ should approach $f_{\infty}$ near the critical point in the following way:

$$
f_{\infty}(t)-f_{N}(t) \sim\left(1 / l^{d}\right) H(l / \xi(t))
$$

$\xi(t)$ is the correlation length of the infinite lattice at temperature $t$. If we replace $f$ by $(8)$ in (7), one finds that $\xi(t)$ and $\xi\left(t^{\prime}\right)$ should be related by the following relationship

$$
\xi(t)=b \xi\left(t^{\prime}\right)
$$

and this is exactly what should give a perfect real space renormalisation for real temperatures. Of course since (8) should be valid only near the ferromagnetic transition point $t_{\mathrm{c}}$ and for large lattices, the renormalisation procedure should in principle become better and better when one increases $N$.

Let us now discuss the case of complex temperatures. The formula (3) contains as an input the partition functions $Z_{N}$ of three lattices of different sizes. Since all these partition functions are polynomials in the variable $t$, the real axis of temperatures does not play any special role. Therefore, we think that there is no reason why (3) should give better results on the real axis than anywhere else in the complex plane of temperatures.

Another attractive aspect of formula (3) is that when applied to hierarchical lattices (Derrida et al 1983), it gives the exact renormalisation transformation for any finite value of $N$. Hence, for hierarchical lattices there is no room for further improvement of the method by increasing $N$.

In order to test the efficiency of the renormalisation method (3), in the remainder of this letter we apply it to the two-dimensional Ising model on the square lattice. For this model $Z_{N}(t)$ is known exactly for any lattice from the Onsager solution

$$
\begin{aligned}
Z_{N}=\frac{1}{2}\left(\frac{2}{1-t^{2}}\right)^{l^{2}}\left\{-\left(1+t^{2}\right)^{2}\left(t^{4}-6 t^{2}+1\right) \prod_{\substack{i, j=1 \\
(i, j) \neq(l / 2, l / 2)}}^{l / 2}\left[A-B\left(\cos \frac{2 i \pi}{l}+\cos \frac{2 j \pi}{l}\right)^{2}\right]\right. \\
+2 \prod_{i, j=1}^{l / 2}\left[A-B\left(\cos \frac{2 i \pi}{l}+\cos \frac{(2 j-1) \pi}{l}\right)^{2}\right] \\
\left.+\prod_{i, j=1}^{l / 2}\left[A-B\left(\cos \frac{(2 i-1) \pi}{l}+\cos \frac{(2 j-1) \pi}{l}\right)^{2}\right]\right\}
\end{aligned}
$$

where $A=\left(1+t^{2}\right)^{4}$ and $B=4 t^{2}\left(1-t^{2}\right)^{2}$. This formula which gives $Z$ as a polynomial in $t$ (except a trivial factor which disappears in formula (3)) can be obtained after some manipulation from the partition function given in McCoy and $\mathrm{Wu}$ (1973). It is valid for a square of even size $l$ with periodic boundary conditions.

We hope in the future to study also other models like the 2D Potts model or 3D Ising model. But for these models, since the partition functions $\mathcal{Z}_{N}$ are not known for arbitrary large $N$, the only hope we can have is that the formula (3) used for $N$ relatively small will give a rather satisfactory picture of the set of singularities in the complex plane. Of course we are conscious that any improvement would require the calculation of the partition function on larger lattices, and we know that this is far from being an easy task. 
Let us now first look at what is the prediction of formula (3) for the location of the critical point $t_{\mathrm{c}}$ and of the thermal exponent $2^{y_{\mathrm{t}}}=\lambda$. For each choice of $N$, formula (3) leads to estimates $t_{\mathrm{c}}^{(N)}$ and $\lambda^{(N)}$ for $t_{\mathrm{c}}$ and $\lambda$. They are obtained by solving numerically the following equation

$$
\frac{Z_{N}\left(t_{\mathrm{c}}^{(N)}\right)}{\left[Z_{N / b^{d}}\left(t_{\mathrm{c}}^{(N)}\right)\right]^{b^{d}}}=\frac{Z_{N / b^{d}}\left(t_{\mathrm{c}}^{(N)}\right)}{\left[Z_{N / b^{2 d}}\left(t_{\mathrm{c}}^{(N)}\right)\right]^{b^{d}}}
$$

and

$$
\lambda^{(N)}=\mathrm{d} t^{\prime} /\left.\mathrm{d} t\right|_{t_{\mathrm{c}}^{(N)}}
$$

where the derivative in (12) can easily be calculated from the implicit function $t \rightarrow t^{\prime}$ defined by (3). Our results for $b=2$ are shown in table 1 and can be compared with the exact values $t_{\mathrm{c}}=\sqrt{2}-1$ and $\lambda=2$.

Table 1. Values of $t_{\mathrm{c}}$ and $\lambda$ obtained from equations (11) and (12) for increasing lattice sizes.

\begin{tabular}{rcll}
\hline$l=N^{1 / d}$ & $N$ & $t_{c}^{(N)}$ & $\lambda^{(N)}$ \\
\hline 8 & $8 \times 8$ & no real fixed point & \\
16 & $16 \times 16$ & 0.418236911 & 1.8505 \\
32 & $32 \times 32$ & 0.414546967 & 1.9781 \\
64 & $64 \times 64$ & 0.414251685 & 1.9951 \\
128 & $128 \times 128$ & 0.414218187 & 1.9988 \\
256 & $256 \times 256$ & 0.414214134 & 1.9997 \\
& & & 2 \\
& Exact value & 0.414213562 & 2 \\
\hline
\end{tabular}

We see that the results converge very well to their expected values. The critical point converges like $1 / l^{3}$ and the thermal exponent like $1 / l^{2}$ so the convergence is as good as the convergence obtained in phenomenological renormalisation (Derrida and De Seze 1982).

We should however point out that for small size $l=8$, there is no fixed point on the real axis.

In principle from (3), one can draw $t^{\prime}$ as a function of $t$ for any choice of $N$ and $b$. In figure 1 , we give the curves obtained for $b=2$ and $N=16^{2}$ and $32^{2}$. It is striking
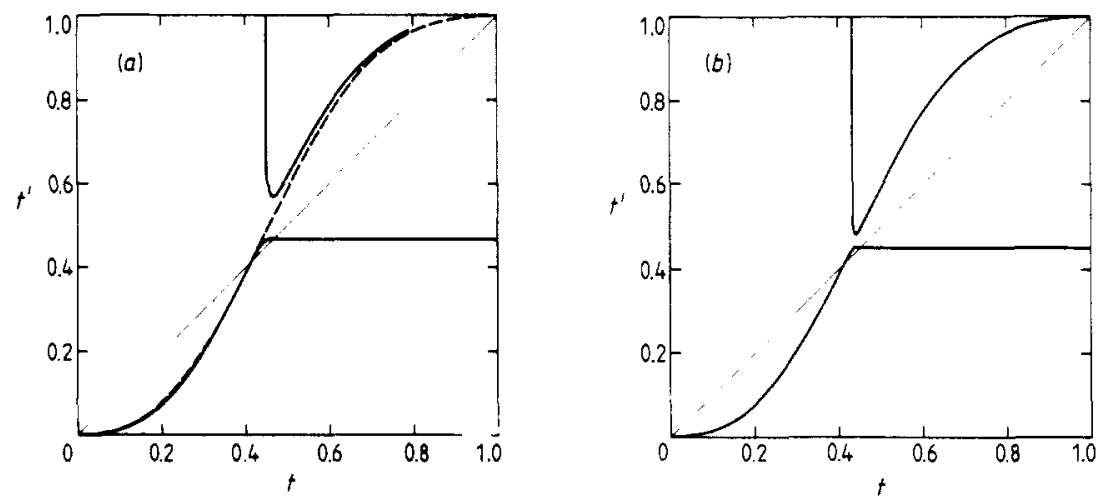

Figure 1. The curves $t \rightarrow t^{\prime}$ obtained from (3) for $b=2$ and $N=16 \times 16$ (figure $1(a)$ ) and $N=32 \times 32$ (figure $1(b)$ ) is shown as full lines. The dashed line in figure $1(a)$ represents the exact real space renormalisation $\xi(t)=2 \xi\left(t^{\prime}\right)$. 
that $t^{\prime}$ is not a single valued function of $t$. This is due to the fact that (3) gives $t^{\prime}$ implicitly. We see also that near the repulsive fixed point, there is an attractive one. The shape of the curve $t \rightarrow t^{\prime}$ has roughly the shape expected, except for its horizontal and vertical branches, the physical origin of which we do not understand. Anyhow, by comparing the curves for $N=16^{2}$ and $32^{2}$ we see that the shape looks more and more what one expects for a good real space renormalisation: two attractive fixed points at $t=0$ and $t=1$ and an unstable fixed point at $t_{\mathrm{c}}$. For comparison we show as a broken line in figure $1(a)$ the function $t \rightarrow t^{\prime}$ obtained from $\xi(t)=2 \xi\left(t^{\prime}\right)$, where $\xi(t)$ is the exact correlation length. This broken line is in principle an exact real space renormalisation. We see that our curves are very close to it; as a matter of fact, in figure $1(b)$ we would not be able to distinguish it from our curves.

We can now look at the shape of the set of singularities in the complex plane which can be obtained from the renormalisation transformation (3).

The main idea to extract the whole set of singularities from the knowledge of a renormalisation transformation $t \rightarrow t^{\prime}$ is that pre-images of singularities must be singularities (Derrida et al 1983). So the set of singularities can be obtained by iterating backwards the renormalisation transformation (see Derrida et al 1983). The way pictures can be produced is by starting with any point $t^{\prime}$ in the complex plane. Then, one finds all the pre-images of $t^{\prime}$ by (3), i.e. all the roots $t$ of equations (3). One chooses one of these roots at iandom, then one calculates all the pre-images of $t$ by (3) and one repeats this procedure again and again. After a few iterations, all the points produced by this method are on the Julia set of the renormalisation transformation.

We show on figure 2 the pictures obtained in the complex plane of the variable $t$ by drawing the Julia set associated with the transformation (3) for several $N=8^{2}$ figure $1(a)$ and $N=16^{2}$ figure $1(b)$. The calculation of these Julia sets is numerically demanding and this is why we did not draw them for larger lattices.

We see that the Julia sets roughly follow the known exact results (the two circles). Moreover, by comparing figures $2(a)$ and $2(b)$, one sees that the points of the Julia set are closer to the two circles for $N=16^{2}$ than for $N=8^{2}$. Therefore we think that increasing $N$ would improve the pictures and in the limit $N \rightarrow \infty$, the Julia set should reduce to the two circles.
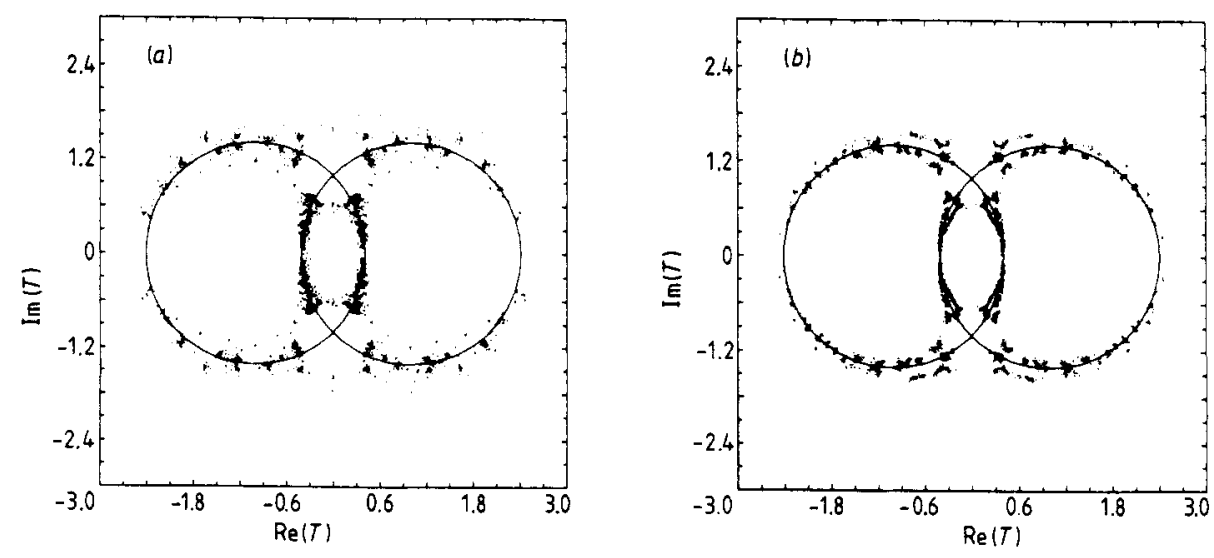

Figure 2. The Julia set of the renormalisation (3) for $b=2$ and $N=8 \times 8$ (figure $2(a)$ ) and $N=16 \times 16$ (figure $2(b)$ ). On each figure the two circles represent the exact known results. 
In this letter we have introduced a new real space renormalisation method which is able to work in the whole complex plane of temperatures. This method requires the knowledge of the partition function of finite lattices of three different sizes. We believe that it should be easy to generalise our calculation to cases where the scaling factor $b$ is not an integer.

It might be a problem to use other boundary conditions than periodic ones because we have seen that to justify (3) one needs finite size scaling and it is not obvious whether with free boundary conditions the finite size scaling would not be buried in surface effects.

It would be interesting to understand the origin of the vertical and horizontal branches that we see on figure 1 , and to know whether they have any physical meaning or are artefacts of our method.

It would be interesting to build other real space renormalisations which would require the knowledge of properties of only two lattices. The reason is that, even if (3) is in principle very simple, the method is very limited by the fact that for most models, the calculation of $Z_{N}$ can only be done for a very few sizes.

Partial support from the French Foreign Ministry and the Danish Research Council is acknowledged by HF.

\section{References}

Barber M N 1983 in Phase Transitions and Critical Phenomena vol 8, ed C Domb and J L Lebowitz (London: Academic)

Burkhardt T W and van Leeuwen J M J 1982 Real space renormalization vol 30 (Berlin: Springer)

Derrida B and De Seze L $1982 J$. Physique 43475

Derrida B, Eckmann J P and Erzan A 1983 J. Phys. A: Math. Gen. 16893

Derrida B, De Seze L and Itzykson C 1983 J. Stat. Phys. 33559

Itzykson C and Luck J M 1984 Proc. Brasov Int. School

Fisher M E 1964 Lectures in Theoretical Physics vol 7c (Boulder: University of Colorado Press) p 1

McCoy B M and Wu T T 1973 The two dimensional Ising Model (Cambridge, Mass.: Harvard University Press)

Nightingale M P 1982 J. Appl. Phys. 537927

Peitgen H O, Prüfer M and Richter P H 1984 Preprint Universität Bremen 